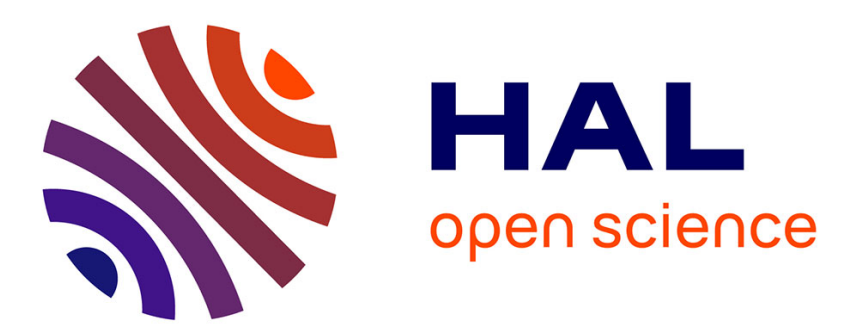

\title{
Meso-scale simulation of liquid feeding in an equiaxed dendritic mushy zone
}

\author{
Yi Feng, Miha Založnik, Brian G. Thomas, André B. Phillion
}

\section{To cite this version:}

Yi Feng, Miha Založnik, Brian G. Thomas, André B. Phillion. Meso-scale simulation of liquid feeding in an equiaxed dendritic mushy zone. Materialia, 2020, 9, pp.100612. 10.1016/j.mtla.2020.100612 . hal-02978787

\section{HAL Id: hal-02978787 \\ https://hal.science/hal-02978787}

Submitted on 23 Dec 2020

HAL is a multi-disciplinary open access archive for the deposit and dissemination of scientific research documents, whether they are published or not. The documents may come from teaching and research institutions in France or abroad, or from public or private research centers.
L'archive ouverte pluridisciplinaire HAL, est destinée au dépôt et à la diffusion de documents scientifiques de niveau recherche, publiés ou non, émanant des établissements d'enseignement et de recherche français ou étrangers, des laboratoires publics ou privés. 


\title{
Meso-scale simulation of liquid feeding in an equiaxed dendritic mushy zone
}

\author{
Y. Feng ${ }^{1}$, M. Založnik², B.G. Thomas ${ }^{3}$, A.B. Phillion ${ }^{1 *}$ \\ ${ }^{1}$ Department of Materials Science and Engineering, McMaster University, Hamilton, Canada \\ ${ }^{2}$ Université de Lorraine, CNRS, IJL, F-54000 Nancy, France \\ ${ }^{3}$ Department of Mechanical Engineering, Colorado School of Mines, Golden, USA
}

\begin{abstract}
A 3D meso-scale model is developed to predict the flow of liquid within a semi-solid binary Fe-C alloy with various equiaxed microstructure, ranging from dendritic to globular. The model domain consists of a set of 8000 grains given by a Voronoi tessellation. Solidification of each grain is simulated independently via a volume average approach, providing the semi-solid microstructure for the fluid flow simulation. A single domain Darcy-Brinkman model is then used to calculate the resulting pressure field.

The model results are found to be in good agreement with the Carman-Kozeny equation for two limiting cases of interfacial area concentration $S_{v}$, demonstrating the model's utility in quantifying permeability of semisolid structures where the fluid flow occurs either in the intra-dendritic (within the envelope enclosed by the dendrite) or extra-dendritic (between dendritic grains) regions. Deviation from Carman-Kozeny behaviour is observed with a transition in microstructure, i.e. when the domain contains a mixture of both dendritic and globular structures or when fluid flow occurs simultaneously in the intra-dendritic and extra-dendritic regions. A permeability-microstructure map is created as a function of grain size, secondary dendrite arm spacing, and cooling rate to show the range where the limiting values of $S_{v}$ are valid and, importantly, where they are not. A comparison of the net volumetric inflow caused by shrinkage and deformation is performed, demonstrating that the shrinkage induced by the peritectic transformation is the dominant factor requiring liquid feeding. The present dendritic fluid flow model is useful in the context of multi-physics modelling of defects in peritectic steel grades and other commercially relevant alloys.
\end{abstract}

\section{Introduction}

Dendrite growth is the most common crystallization mechanism observed during continuous casting of steel.

4 The morphology characterized by the dendrite arms is associated with the formation of secondary phases and cast6 ing defects, most notably hot tearing, porosity and segregation [1, 2]. Advanced continuously-cast high strength ${ }_{32}$ steel slabs with high levels of alloying elements as well as complex shape castings are quite prone to these defect.

10 The formation of casting defects, especially hot tearing, is a multi-scale problem, and has been shown to be related ${ }_{36}$ directly to the flow of liquid through the dendritic network at the microscale [3], due to the concomitant phenomena

14 of solidification induced shrinkage and mushy zone deformation.

The resistance to liquid flow through a semisolid is known as permeability. This important macroscopic pa- ${ }_{42}$ 18 rameter is associated with a pressure drop inside the mushy zone of a casting. It bridges the microscale structure with macroscale fluid flow, and is critical for accurate prediction of defect formation. Permeability was initially proposed ${ }_{46}$ 2 for transport phenomena in porous media, and has been extensively investigated in various material systems. Mea24 surement of permeability is usually associated with deter- mination of the structure first followed by a prediction of the fluid flow behaviour [4. The challenge when measuring this quantity in metallic systems lies in controlling the semisolid microstructure during the experiment; reliable data for high temperature alloys remains rare [5]. Despite the recent application of X-ray tomography in obtaining the complicated topological images for use as templates to construct physical models of the dendritic structure [4, the accessibility of accurate experimental apparatuses is limited 6.

Predictive numerical simulation is a well-studied alternative to experimental investigation of permeability and phenomenological models as detailed in Refs. [5, 7, 8, 9, 10. Numerical models solve the Stokes equations for a domain representing the liquid phase within the mushy zone. The obvious advantage of using a simulated microstructure is that the evolution of permeability with solid fraction can be easily studied for different grain sizes and morphologies. However, the main challenge with this method is the geometry itself, since permeability is a characteristic that is based on the channel width, surface area and tortuosity of the flow channels 8. Recently, 3D synchrotron $\mathrm{X}$-ray tomography has been used to acquire representative semisolid geometries for assessing permeability during 
equiaxed dendritic 5] and columnar solidification [10,104 investigate the effects of intermetallics 11. Although these studies have shown good agreement between 106 calculated permeability and experimental reference data, the availability of high-quality $3 \mathrm{D}$ datasets has limited the 108 54 use of this approach to well defined systems. For industrial applications, there is a need for improved understanding ${ }_{110}$ 56 of permeability in a wide range of microstructures.

The recent development of a meso-scale granular model ${ }_{112}$ within a domain. The grains are individually represented ${ }_{116}$ 62 and the flow of liquid between the grains is simulated. A 2D discrete-element model of fluid flow proposed by ${ }_{118}$ 64 Vernède et al. [14 described the liquid feeding along the grain boundaries of the mushy zone. This approach was

${ }_{66}$ then extended to 3D by Sistaninia et al. [15], and further modified by Zareie-Rajani and Phillion [16] to investigate ${ }_{68}$ phenomena relevant to hot tearing in aluminum alloys during welding. These prior granular solidification models 70 assumed a microstructure consisting of globular equiaxed grains, and modelled the flow between adjacent grain sur-

72 faces as Poiseuille flow between two parallel plates. Because of these limitations, they cannot be used to assess the permeability of a dendritic microstructure. In the dendritic case, liquid flow occurs both in the extra76 dendritic region between grains as well as in the intradendritic small-scale structure within a grain. The fric-

78 tion encountered by the flow through intra-dendritic liquid channels could lead to an additional pressure drop thus accelerating the formation of defects.

In the present study, a $3 \mathrm{D}$ meso-scale model is pro- ${ }_{120}$ 2 posed to simulate fluid flow during the solidification of a binary $\mathrm{Fe}-\mathrm{C}$ alloy mushy zone containing both intra84 dendritic and extra-dendritic flow and taking into account ${ }^{122}$ shrinkage caused by the peritectic transformation and de-

86 formation. The meso-scale simulation domain is created $^{124}$ using a Voronoi tessellation; solidification of each grain oc-

${ }_{88}$ curs independently via a volume average approach [17, 18..$^{126}$ First, the methodology for creating a semi-solid domain 90 consisting of equiaxed-dendritic grains surrounded by liq- ${ }^{128}$ uid is reviewed. Second, the new liquid flow model is de-

92 scribed. Third, the model is applied to investigate permeability in a wide combination of microstructures, alloy

94 compositions and flow configurations during solidification in order to create a map of the resulting permeability. Fi96 nally, the results are compared with predictions of previ- ${ }^{134}$ ous simple models and assessed in the context of casting 98 defects.

2. Description of the 3D Meso-scale Fluid Flow Model for Dendritic Alloys

\subsection{Generation of the Model Domain}

In order to investigate the effects of fluid flow within ${ }^{142}$ the semisolid Fe-C alloy, a model domain must be uti- lized that is large enough to capture long-range flow effects while small enough to discretize individual grains - in order words - a domain that contains hundreds (or more) grains. This study utilizes the 3D equiaxed-dendritic meso-scale solidification model previously described in [19] to create an appropriately-sized model domain. An example, containing 1000 grains, is shown in Fig. 11(a); the empty space within the domain represents the remaining liquid. To create this geometry, a Voronoi tessellation is applied to approximate the final grain morphology based on randomlyplaced seeds acting as nuclei for equiaxed-dendritic grains. Each grain, Fig. 1 (b), is a polyhedral structure, which is then divided into smaller polyhedrons, Fig. 1 (c), and finally a tetrahedron, Fig. 1(d). Solidification is simulated within each tetrahedron independently of all others, as described below.

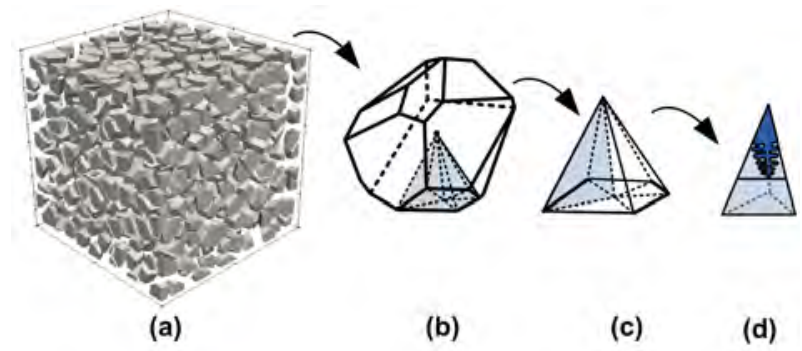

Figure 1: (a) Meso-scale simulation domain containing 1000 grains; (b) Single Voronoi grain; (c) Polyhedral structure; (d) Tetrahedron with an illustrative schematic of the equiaxed-dendritic microstructure.

\subsection{Dendritic Solidification Model}

Although the complete details of the 3D meso-scale dendritic solidification model are given in [19], the salient points are recalled below for clarity. Given that the microstructure of steels is dendritic, the evolution in solid fraction within each tetrahedron is predicted using a volume average approach [17, 18. The main advantage of this approach is its ability to model solidification using at least three and possibly four phases, extra-dendritic liquid $l^{e d}$, intra-dendritic liquid $l^{i d}$, delta-ferrite $\delta$ and austenite $\gamma$, without explicitly tracking the interfaces between the phases. The $\delta$ and/or $\gamma$ phases nucleate from one vertex of the tetrahedron corresponding to the center of the grain in an undercooled liquid and grow in a radial direction until the tetrahedron is fully solidified. The entire tetrahedron is considered to be at uniform temperature. A dendritic grain is defined by its envelope, which controls the solid $(\delta$ and $\gamma$ ) phases, the intra-dendritic liquid phase (i.e. the liquid enclosed by the dendrite envelope), and the extra-dendritic liquid phase (i.e. the liquid outside the dendrite envelope). The phases are described by their volume fractions $\left(g_{l}^{e d}, g_{l}^{i d}, g_{\delta}\right.$ and $\left.g_{\gamma}\right)$ and average chemical compositions. Upon cooling a solute mass balance is performed to track the position of the dendrite envelope 


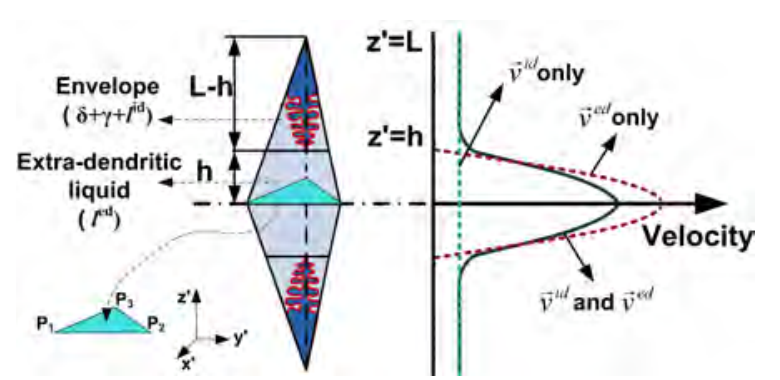

Figure 2: Schematic diagram of two facing tetrahedrons, the velocity profile of fluid passing through the inter- and extra- dendritic regions, and the corresponding 3 -node $2 \mathrm{D}$ triangular element. The velocity profiles for the cases with only intra-dendritic and only extradendritic flow are also shown.

The flow in the extra-dendritic region is described as a

under the assumption of finite diffusion in $l^{e d}$, $l^{i d}$, and $\delta$ or $\gamma$. Note that the dendritic morphology indicated in ${ }_{184}$ ig. 1(d) cannot be visualized by the unstructured mesh given in Fig. 1(a); this image shows only the equivalent ${ }_{186}$ solid phase fraction in a geometric sense with the empty space including both the intra- and extra- dendritic liquid.188

\subsection{Mushy zone fluid flow model}

The 3D semisolid structure created by the solidification model at a given solid fraction for a specified cooling rate and grain size is used as the input geometry for the fluid flow model at the same solid fraction. The two models, solidification and fluid flow, are only one-way coupled. The mesh consists of a set of elements, each made ${ }_{190}$ up of two facing tetrahedrons as shown in Fig. 2, that are ultimately reduced to a set of two 3-node 2D trian- 192 gular elements. The regions enclosed by the dendrite envelopes of each tetrahedron are treated as a uniform porous ${ }_{194}$ medium [20] with an internal liquid fraction given by $g_{l}^{\prime}=$ $g_{l}^{i d} /\left(g_{l}^{i d}+g_{\delta}+g_{\gamma}\right)$. The extra-dendritic regions of each $_{196}$ element, having a width equal to the distance between the facing envelopes, are treated as an extra-dendritic fluid channel. Note that the two facing tetrahedrons are identical due to symmetry [15]. Flow can occur simultaneously through both the intra- and extra- dendritic regions as shown schematically in Fig. 2 (solid blue line). In the limit of $g_{l}^{\prime}=0$, the model is reduced to the model of flow between two globular grains, equivalent to the model ${ }^{200}$ of Sistaninia 15. In another limit, where the dendrite tips touch and all remaining liquid is intra-dendritic liq- ${ }^{202}$ uid $\left(g_{l}^{\text {ed }}=0, g_{l}^{i d}=g_{l}^{\prime}\right)$, the whole structure behaves as a porous medium with a liquid fraction $g_{l}^{\prime}$ and a characteristic length scale given by the secondary dendrite arm spacing. In these two situations, also shown in Fig. 2 the corresponding flow is either Poiseuille flow (red dashed line) or Darcy-Brinkman flow (green dashed line). Poisseuille flow and the flow in the intra-dendritic region is described by the Darcy-Brinkman equation, using a av-214 eraged form of the Navier-Stokes equation. The model assumptions include quasi-steady-state as well as irrotational flow that is parallel to the triangular facet highlighted in blue in Fig. 2 where the two tetrahedrons meet. Both gravity and pressure gradients along the length $L$ of the element are neglected. Altogether, this is expressed as,

$$
\begin{gathered}
-\nabla p+\mu_{l} \frac{d^{2} \vec{v}^{e d}}{d z^{2}}=0, \\
-g_{l}^{\prime} \nabla p+\mu_{l} \frac{d^{2} \vec{v}^{i d}}{d z^{\prime 2}}-\frac{\mu_{l} g_{l}^{\prime}}{K\left(g_{l}^{\prime}\right)} \vec{v}^{i d}=0,
\end{gathered}
$$

where $\mu_{l}$ represents the dynamic viscosity, $\vec{v}^{e d}$ is the fluid velocity in the extra-dendritic region, $p$ is the gauge pressure, $\vec{v}^{i d}$ is the intra-dendritic fluid velocity vector and $K\left(g_{l}^{\prime}\right)$ is the local permeability within the dendrite envelope. The reader is referred to 21] for detailed information of the averaging concepts along with the process of deriving the average form of the master equation. The Carman-Kozeny equation 22, 23],

$$
K\left(g_{l}^{\prime}\right)=\frac{\left(g_{l}^{\prime}\right)^{3}}{5 S_{v}^{2}}
$$

where $S_{v}$ represents the interfacial area concentration is simplified as $S_{v}=\frac{2}{\lambda_{2}}$ with $\lambda_{2}$ representing the secondary dendrite arm spacing, is used to determine $K$ as the scale of an individual element.

We assume that $\left.\frac{\partial}{\partial z^{\prime}} \vec{v}^{e d}\right|_{z^{\prime}=0}=0$ and $\vec{v}$ is finite when $z^{\prime} \rightarrow \infty$. At the envelope we use the boundary conditions between the porous medium and a fully liquid zone, proposed by Le Bars and Grae Worster 21]: $\left.\vec{v}^{e d}\right|_{z^{\prime}=h}=$ $\left.\vec{v}^{i d}\right|_{z^{\prime}=h}$ and $\left.\frac{\partial}{\partial z^{\prime}} \vec{v}^{i d}\right|_{z^{\prime}=h}=\left.\frac{\partial}{\partial z^{\prime}} \vec{v}^{e d}\right|_{z^{\prime}=h}$. Eq. 1 and Eq. 2 can be solved analytically,

$$
\begin{aligned}
\vec{v}^{e d} & =\left(\frac{z^{\prime 2}}{2 \mu_{l}}+C_{1}\right) \nabla p, \\
\vec{v}^{i d} & =\left(C_{2} e^{\frac{z^{\prime}}{\xi}}-C_{3}\right) \nabla p .
\end{aligned}
$$

In Eqs. 4 and 5. $C_{1}$ and $C_{2}$ represent two unknown constants, $C_{3}=\frac{g_{l^{\prime}} \xi^{2}}{\mu_{l}}$ and $\xi=\sqrt{\frac{K\left(g_{l}^{\prime}\right)}{g_{l}^{\prime}}}$. The unknown constants can be further solved with additional constraints at the envelope shown above: all of the fields within the representative volume are continuous, the velocity $\vec{v}$ and the viscous stress at the interface between the intra-dendritic and extra-dendritic regions must be continuous. Then,

$$
\begin{gathered}
C_{1}=-\frac{\xi h}{\mu_{l}}-\frac{g_{l}^{\prime} \xi^{2}}{\mu_{l}}-\frac{h^{2}}{2 \mu_{l}}, \text { and }, \\
C_{2}=\frac{-\frac{\xi h}{\mu_{l}}}{e^{-\frac{h}{\xi}}}
\end{gathered}
$$

where $h$ is the half width of the extra-dendritic region.

The controlling equation for the fluid flow problem can be derived through integration based on a mass balance 
over the two facing tetrahedrons shown in Fig. 2 assuming

liquid incompressibility, i.e. $\nabla \cdot \vec{v}_{l}=0$. This region includes both flow as a porous medium within the dendrite envelope and free liquid flow in the extra-dendritic region. This mass balance also needs to consider both the solidification shrinkage and deformation as factors that would induce liquid flow. The shrinkage induced by solidification due to the density variations in the solid and liquid phase will induce a normal velocity of liquid flow at the solid/liquid interface [24],

$$
\vec{v}_{l \cdot n}=-\beta v^{*},
$$

where $v^{*}$ is the solid/liquid interface velocity predicted by the $3 \mathrm{D}$ meso-scale solidification model at the specific solid fraction being used in the fluid flow simulation, and $\beta=\left(\rho_{s} / \rho_{l}-1\right)$ is the shrinkage factor with $\rho_{s}$ and $\rho_{l}$ representing the temperature-dependent solid and liquid densities. For non-peritectic alloys, $\rho_{s}=\rho_{\delta}$. For low carbon steel alloys having a peritectic transformation, $\rho_{s}$ is given by

$$
\begin{gathered}
\rho_{s}=\frac{\rho_{\delta} g_{\delta}+\rho_{\gamma} g_{\gamma}}{g_{\delta}+g_{\gamma}}, \\
\rho_{\delta}=3.07 \times 10^{-1}\left(T_{\delta, \text { start }}-T\right)+7270, \\
\rho_{\gamma}=4.8 \times 10^{-1}\left(T_{\gamma, \text { start }}-T\right)+7410, \\
\rho_{l}=-7.5 \times 10^{-1}\left(T-T_{L, \text { start }}\right)+7020,
\end{gathered}
$$

where $\rho_{\delta}$, and $\rho_{\gamma}$ represent the densities $\left(\mathrm{kg} \cdot \mathrm{m}^{-3}\right)$ of the $\delta$ and $\gamma$ phases given by the expressions in Eqs. $10,11,12$ with different coefficients $\left(\mathrm{kg} \cdot \mathrm{m}^{-3} \cdot \mathrm{K}^{-1}\right)$ [25], $T$ represents ${ }_{268}$ the temperature $(\mathrm{K})$ with $T_{i, \text { start }}$ being the transformation temperatures of the $i$ phase $(i=l, \delta$ or $\gamma)$, and $g_{\delta}$ and $g_{\gamma}$ are given by the 3D meso-scale solidification model at the specified solid fraction being used in the fluid flow simulation. Note that the shrinkage factor will vary during solidification since the individual densities $\rho_{\delta}, \rho_{\gamma}$, and $\rho_{l 270}$ are temperature-dependent.

Deformation of the semi-solid skeleton will also induce 272 liquid flow. Assuming rigid body motion of the grains and deformation localized to the liquid phase, the increase in $_{274}$ volumetric flow rate $\Delta v_{l i q}$ that is required to compensate for deformation at the scale of an individual element can be approximated as

$$
\Delta v_{l i q}=\frac{\dot{\varepsilon}_{s v}}{\left(1-g_{s}\right)} * V_{l i q}
$$

where $\dot{\varepsilon}_{s v}$ is the volumetric part of the strain rate applied on the domain, and calculated via $\dot{\varepsilon}_{s v}=\dot{\varepsilon}_{x x}+\dot{\varepsilon}_{y y}+_{278}$ $\dot{\varepsilon}_{z z}$, and $V_{l i q}$ represents the volume of liquid present in an element. Note that while Eq. 13 simulated the effects ${ }_{280}$ of mechanical deformation on fluid flow in a semi-solid, mechanical deformation itself is not directly simulated.

Applying the divergence theorem, the mass balance becomes

$$
\int_{V_{l}^{e}} \nabla \cdot \vec{v}_{l} d V=2 \cdot \int_{S_{s l}^{e}} \vec{v}_{l} \cdot \vec{n} d S+2 \cdot \int_{S_{l}^{e}} \vec{v}_{l} \cdot \vec{n} d S+2 \cdot \Delta v_{l i q}=0,
$$

where $V_{l}^{e}$ represents the total volume of the two facing tetrahendrons, $S_{s l}^{e}=S_{v} \cdot V^{e}$ is the dendritic solid/liquid interfacial area, $V^{e}$ represents the total volume of dendrite envelope, and $S_{l}^{e}$ represents the total lateral area of the two tetrahedral elements. Then, by substituting Eq. (4) and Eq. (5) into the second right term of Eq. (14), and assuming that the first right term of Eq. (14) can be replaced by $S_{s l}^{e} \cdot \vec{v}_{l \cdot n}=-S_{s l}^{e} \cdot \beta v^{*}$, one obtains the master fluid flow equation for dendritic flow,

$$
\begin{aligned}
2 \int_{S_{l}^{e}}\left(\frac{z^{\prime 2}}{2 \mu_{l}}+C_{1}\right) \nabla p \cdot \vec{n} & d S+2 \int_{S_{l}^{e}}\left(C_{2} e^{\frac{z^{\prime}}{\delta}}-C_{3}\right) \nabla p \cdot \vec{n} d S \\
- & 2 v^{*} \beta S_{s l}^{e}+2 \cdot \Delta v_{l i q}=0 .
\end{aligned}
$$

\subsection{Numerical implementation of fluid flow model}

258 over the intra-dendritic and extra-dendritic pa puted numerically by dividing both the grain envelope length and extra-dendritic liquid channel width into $n=$ 1000 segments along the height of tetrahedral element. By doing the integration and applying Green's theorem over each segment, one obtains the coefficient of the Laplacian of the pressure field, $\nabla^{2} p$. Then, as it has been assumed that the flow direction is parallel to the exterior triangular facet of each tetrahedron, the 3D mesh is simplified to a set of 3 -node $2 \mathrm{D}$ triangular elements. The resulting pressure field is given by

$$
p_{l}=\sum_{i=1}^{3} N_{i} p_{i}^{*}
$$

where $p_{i}^{*}$ represents the nodal pressures, and $N_{i}$ represents the shape functions of the triangular element that approximate the pressure field within element $e$ in the local $\left(x^{\prime}, y^{\prime}, z^{\prime}\right)$ coordinate system. Applying the Galerkin finite element method to Eq. (15), the elemental matrix equation is obtained:

$$
[K]^{e}\left\{\begin{array}{c}
p_{1}^{*} \\
p_{2}^{*} \\
p_{3}^{*}
\end{array}\right\}=b^{e}+\{\phi\}^{e}
$$

where $[K]^{e}$ represents the element stiffness matrix, $b^{e}$ is the load vector which results from solidification shrinkage and/or deformations exerted on the domain, and $\{\phi\}^{e}$ is related to the external boundary conditions.

Once the individual element matrices have been developed, they are assembled together into the global stiffness matrix. This global matrix is then solved with a conjugate gradient linear iterative method using a free open access 


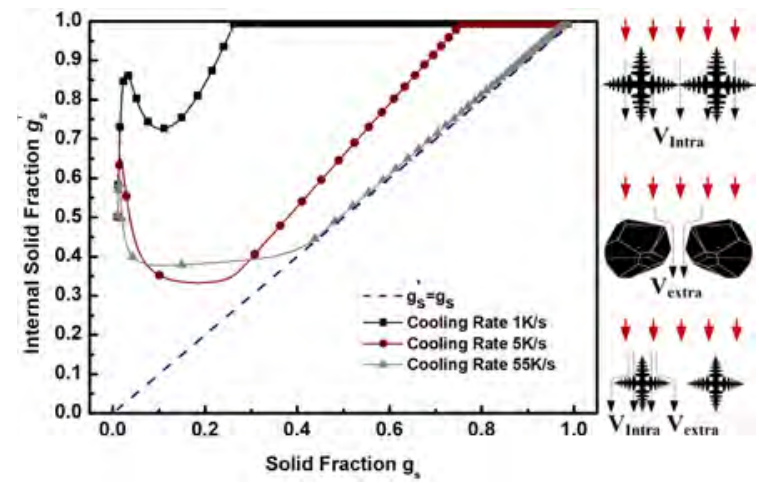

Figure 3: Internal solid fraction evolution within a single grain with ${ }^{352}$ a final diameter of $300 \mu \mathrm{m}$ under three cooling rates along with the schematic diagrams of intra-dendritic, extra-dendritic and both fluid ${ }_{354}$ flow types. The dashed line represents the curve $g_{s}^{\prime}=g_{s}$.

At the highest cooling rate of $55 \mathrm{~K} / \mathrm{s}$, the solidification ${ }^{350}$ model predicts a semisolid structure where the dendrite tips touch each other at a solid fraction of 0.45 (i.e where this curve intersects the $g_{s}^{\prime}=g_{s}$ curve). Beyond this solid fraction all the liquid is intra-dendritic, and thus the flow would also be intra-dendritic as illustrated in the "upper right" diagram of Fig. 3. As $g_{s}$ increases, the permeability of the porous medium would correspondingly be reduced. For the low cooling rate of $1 \mathrm{~K} / \mathrm{s}$, the grain morphology transitions from dendritic to globular at $g_{s}=0.22$ as $g_{s}^{\prime} \rightarrow 1$. It is at this point that the existing dendrite structure becomes fully solid; the remaining extra-dendritic liquid within the element then solidifies in globular fashion. In a globular grain morphology, the permeability within the dendrite envelope is zero, and fluid flow will only take place in the extra-dendritic region as shown in the "middle right" diagram. At moderate cooling rates, both intradendritic and extra-dendritic flow can take place as shown in the "lower right" diagram since the grain is dendritic yet the dendrites from adjoining grains have not yet touched. In the case of $5 \mathrm{~K} / \mathrm{s}$, this flow pattern is possible until program $\mathrm{C}++$ library known as IML ++26 . The solution provides the pressure throughout the domain. Com-322 plete details of the numerical implementation can be found in 15.

\section{Results and Discussions}

To study liquid feeding within a semisolid, the mi-328 crostructure and the solid fraction of individual grain needs to be determined first; the local solid fraction predicted by ${ }^{330}$ the solidification model [19] provides both the local permeability at the grain scale through Eq. 3 and the extra-332 dendritic liquid channel width.

Fig. 3 shows the evolution in internal solid fraction ${ }^{334}$ $\left(g_{s}^{\prime}=1-g_{l}^{\prime}\right)$ given by the solidification model under three cooling rates $(1,5$, and $55 \mathrm{~K} / \mathrm{s})$ for an Fe- $0.07 \mathrm{wt}^{\circ} \% \mathrm{C}$ grain $^{336}$ with a final diameter of $300 \mu \mathrm{m}$, along with schematics of the corresponding flow patterns. $g_{s} \sim 0.75$ at which point the flow would become extradendritic since $g_{s}^{\prime} \rightarrow 1$.

The flow patterns qualitatively described in Fig. 3 can be quantitatively described using the $3 \mathrm{D}$ fluid flow model. For these simulations, a domain $6 \times 6 \times 6=216 \mathrm{~mm}^{3}$ with 8000 cubic grains each $300 \mu \mathrm{m}$ in equivalent diameter ( $d=\sqrt[3]{V_{g}}$ with $V_{g}$ being the grain volume), assuming a dynamic viscosity of $\mu_{l}=7.0 \times 10^{-3} \mathrm{~Pa} \cdot \mathrm{s}$ [27, and neglecting solidification shrinkage and deformation $\left(\beta=0, \dot{\varepsilon}_{s v}=0\right)$ was utilized. The secondary arm spacing was kept $\lambda_{2}=20 \mu \mathrm{m}$. The boundary conditions were set as follows: a constant pressure on the top surface where the fluid is drawn in, i.e. $p_{0}=0 \mathrm{~Pa}$, a constant non-zero average flux on the bottom surface of $-20 \mathrm{~m}^{3} / \mathrm{\mu m}^{2} \cdot \mathrm{s}^{-1}$ and closed lateral boundaries, i.e. $q_{l}=0 \mathrm{\mu m}^{3} / \mathrm{\mu m}^{2} \cdot \mathrm{s}^{-1}$. Due to non-zero fluid flux on the bottom surface and closed lateral surfaces, downward flow inside the domain occurs, drawing fluid in from the top surface.

Fig. 4 shows pressure maps for three semisolids, each at $g_{s}=0.60$, containing cubic equiaxed grains created under different cooling rates $(1,5$, and $55 \mathrm{~K} / \mathrm{s})$. The results provide a general view of the pressure distribution and the different pressure drops resulting from different microstructures. The pressure is seen to decrease almost linearly from the top to the bottom, indicating that the further away from the top of the domain the lower pressure is. A significant pressure drop is observed with the high cooling rate of $55 \mathrm{~K} / \mathrm{s}$ (Fig. 4(c)), achieving a local value of -986 $\mathrm{Pa}$. This is an indication that a higher resistance of liquid is found when liquid going through an intra-dendritic network as compared to globular structure (Fig. 4(a)), where free fluid flow occurs only in the extra-dendritic zone. Between the two extreme cases, the presence of the liquid in both extra-dendritic and intra-dendritic region leads to an intermediate pressure drop (Fig. 4(b)). Although it is unrealistic to assume a constant grain size (the same equivalent grain diameter) for different cooling rates, we have done this for the purpose of decoupling cooling rate and grain size effects as they relate to flow behaviour. As the fully solidified structure is generated using a Voronoi tessellation, the average grain size could be varied as a function of cooling rate based on an empirical equation; however grain size measurement of primary delta grains are very challenging because of the $\delta$ to $\gamma$ solid state phase transformation.

\subsection{Permeability assessment}

\subsubsection{Limiting cases and transition tested}

The dendritic fluid flow model can be verified by comparing its predictions of permeability against corresponding predictions from the analytical Carman-Kozeny equation for two scenarios: a dendritic structure with $S_{v}=$ $\frac{2}{\lambda_{2}}$ [20] (termed Dendritic $S_{v}$ ) and a globular structure with $S_{v}$ calculated as the sum of the grain surface areas assuming globular structure divided by the volume of the whole domain [15] (termed Globular $S_{v}$ ). For these tests, a 

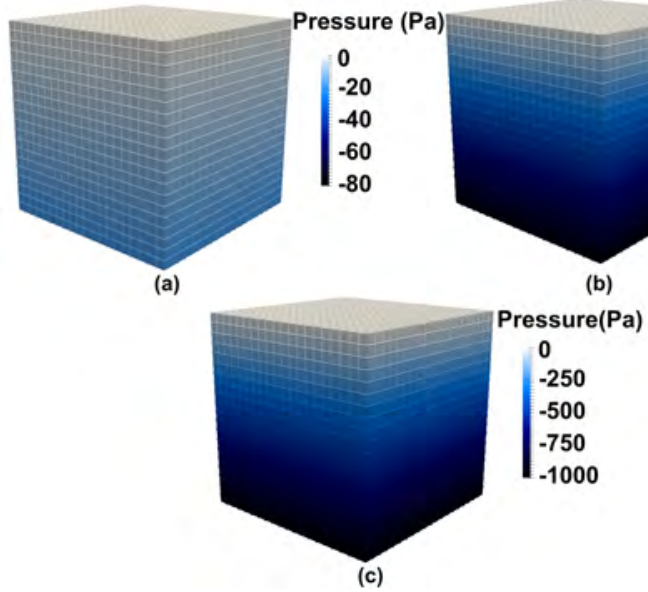

Figure 4: Pressure distribution within a domain containing 8000 grains at $g_{s}=0.60$ solidified under three cooling rates: (a) $\mathrm{CR}=1 \mathrm{~K} / \mathrm{s}$, (b) $\mathrm{CR}=5 \mathrm{~K} / \mathrm{s}$ and (c) $\mathrm{CR}=55 \mathrm{~K} / \mathrm{s}$. Note that Fig. 4(a) and (b) share the same color bar.

series of simulations were performed between $0.5<g_{s}<1$ under the conditions described previously using uniform cubic grains. The permeabilities predicted by the simulations can then be calculated from the average pressure difference between the top and bottom surfaces,

$$
K=\mu_{l} \frac{q_{1}}{\left(\frac{p_{1}-p_{0}}{L_{d i s}}\right)},
$$

where $L_{d i s}$ is the distance between the two surfaces, $p_{1}$ is the averaged pressure on the bottom side of the domain,400 and $q_{1}$ is the flux on the bottom surface. Note that as there is a single grain size and a uniform temperature ap-408 plied to the entire domain, there can be no variation in the permeability between individual cubic grains.

Fig. 5 compares the permeability predicted by the 3D fluid flow model and the values calculated with the Carman ${ }_{* 12}$ Kozeny equation utilizing the Dendritic $S_{v}$ and the Globular $S_{v}$. As can be seen, an excellent match is achieved $4_{414}$ between the simulations with a cooling rate of $55 \mathrm{~K} / \mathrm{s}$ (green diamonds) and the dendritic-flow analytical solu-416 tion. In this scenario, $\vec{v}^{\text {ed }}$ is zero and the domain is a porous medium with a uniform $\vec{v}^{i d}$ flowing through the 418 intra-dendritic regions. Further, an excellent match is achieved between the simulations with a cooling rate of $1_{420}$ $\mathrm{K} / \mathrm{s}$ (red circles) and the globular-flow analytical solution.

In this scenario, flow occurs only in the extra-dendritic422 regions.

The interesting result occurs for the permeabilities cal-424 culated from the simulation using a cooling rate of $5 \mathrm{~K} / \mathrm{s}$ (blue triangles). As can be seen, there is a significant de-426 viation between the model's predictions and the CarmanKozeny equation using the two limiting values for $S_{v}$ up to428 a solid fraction of $\approx 0.75$. Initially, the dendrite envelope grows into the liquid and $g_{s}^{\prime}$ is relatively low (Fig. 3). Fluid $\mathrm{d}_{430}$ thus flows through both the intra-dendritic and extra-dendritic regions, causing the permeability to fall between the den-432 dritic and globular cases. As $g_{s}^{\prime} \rightarrow 1$, flow becomes predominantly extra-dendritic and eventually the permeability follows the Carman-Kozeny equation derived based on the Globular $S_{v}$.

By testing the numerical results against an analytical equation, the present model is shown to be an alternative technique for obtaining the semisolid permeability. The calculated values could also be compared to experimental measurement using the given interfacial surface area concentration to provide additional insight.

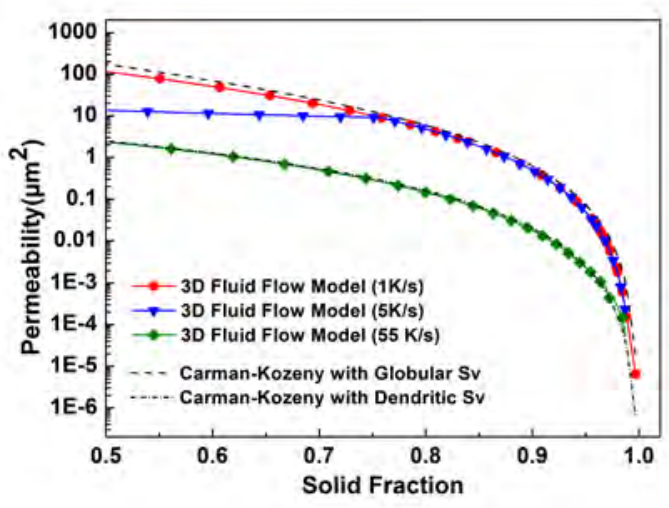

Figure 5: Validation of permeability predicted by present model with the Carman-Kozeny equation for a uniform network of grains with microstructure solidified under the cooling rate of $1 \mathrm{~K} / \mathrm{s}, 5 \mathrm{~K} / \mathrm{s}$ and $55 \mathrm{~K} / \mathrm{s}$.

\subsubsection{Influence of grain size on the permeability}

The assumption made in Fig. 5 was of uniform grain size. However, this is not a realistic description of microstructure. Fig. 6(a) shows the relative frequency of grain size in a $3 \mathrm{D}$ domain created using the Voronoi tessellation for an average grain size of $300 \mu \mathrm{m}$. Fig. 6(b) shows the corresponding evolution in $g_{s}^{\prime}$ for five different grain sizes each solidified using a cooling rate of $5 \mathrm{~K} / \mathrm{s}$. As can be seen, for smaller grains $(60 \mu \mathrm{m}), g_{s}^{\prime}$ quickly approaches 1 and thus forms a globular structure due to the constraints of solute enrichment in front of the solid/liquid interface, whereas for coarse grains $(722 \mu \mathrm{m})$ the dendrite tip is free to move until impingement with neighbouring grains. Thus, at a specific time, which corresponds to a specific bulk solid fraction, fluid flow takes place in the extra-dendritic region for smaller realistic grains, passes through the intra-dendritic region for these grains which are impinging with their neighbours, and has mixed characteristics for grains at intermediate size levels.

Given the intrinsic variability in grain size, the permeability within a semisolid will be influenced by this quantity. Fig. 7 shows the permeability within a domain with 8000 grains, having an average grain size of $300 \mu \mathrm{m}$, predicted by the fluid flow model containing a mixture of both globular and dendritic grains of realistic geometry. It can be seen that at lower solid fraction, the permeability of the mushy zone neither follows the intra-dendritic 


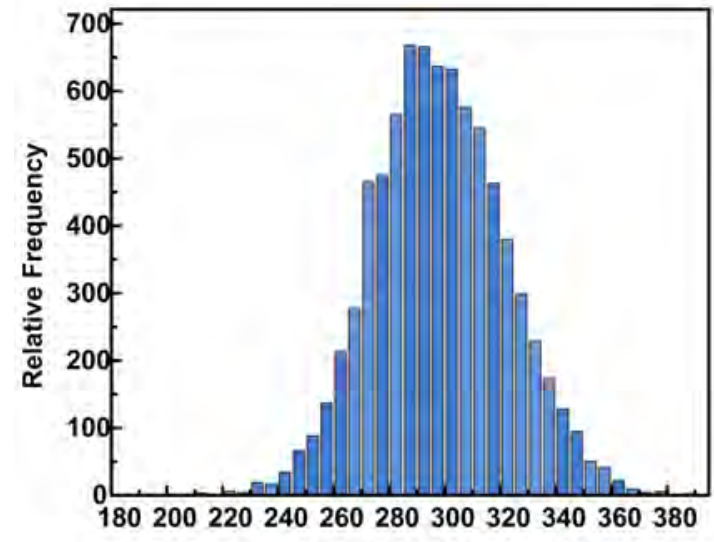

(a) Equivalent Grain Size $d(\mu \mathrm{m})$

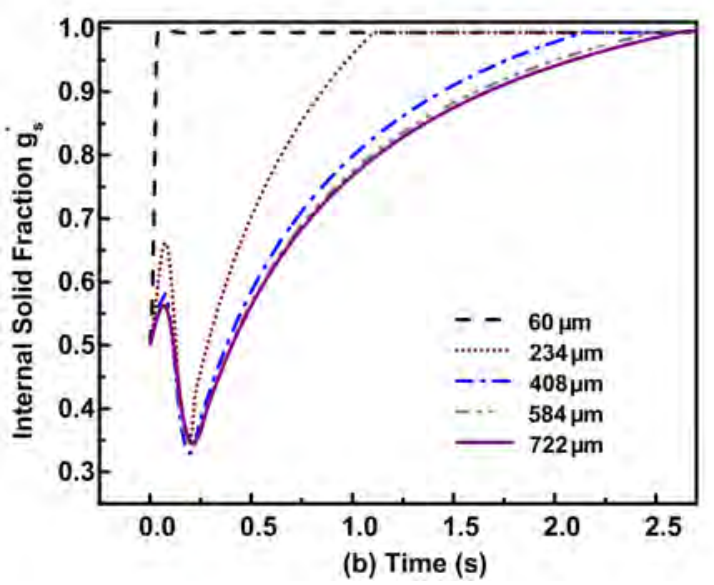

Figure 6: (a) Equivalent grain size $d$ distribution within the semisolid domain and (b) the variation in $g_{s}^{\prime}$ for five grains containing different sizes.

flow behaviour (Carman-Kozeny with Dendritic $S_{v}$ ) nor the extra-dendritic flow behaviour (Carman-Kozeny with470 Globular $S_{v}$ ) but is a mixture of both. Eventually, the permeability approaches the Carman-Kozeny permeabil-472 ity for structure with Globular $S_{v}$. In this realistic case the Carman-Kozeny equation with Dendritic $S_{v}$ does not474 provide a good analytical description of permeability until $g_{s}=0.96$

\subsubsection{Permeability-Microstructure Map}

Referring to Fig. 7) it can be seen that the semisolid permeability can only be predicted by the Carman-Kozeny480 equation with Dendritic $S_{v}$ or Globular $S_{v}$ over a small range of solid fraction; outside of this range there is $a^{482}$ great deviation from either the dendritic or the globular cases. This deviation has not been identified before. In $^{484}$ order to show the range of validity of the Carman-Kozeny equation using these two limiting cases in predicting the ${ }^{486}$ mushy zone permeabilities in metallic alloys, a series of quasi-steady flow simulations were performed, by varying 488 the solid fraction (30 values), cooling rate (10 values assuming an average grain size of $300 \mu \mathrm{m}$ and a secondary490

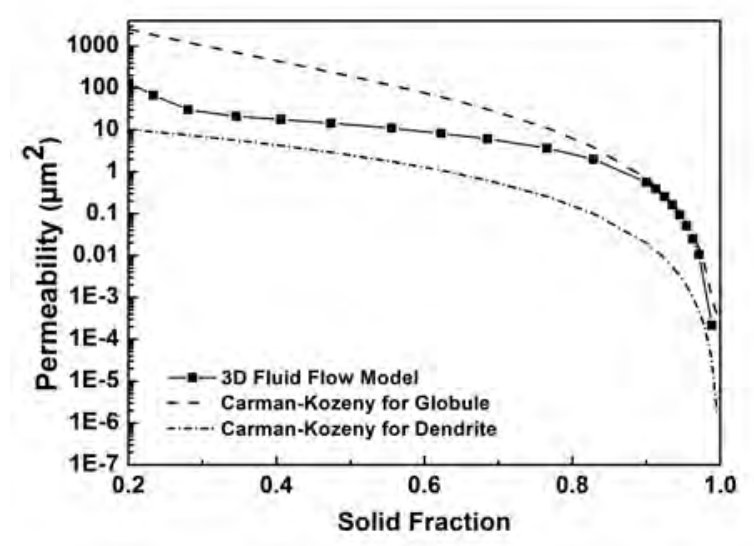

Figure 7: Variations of permeability as a function of solid fraction for a semisolid domain containing both intra-dendritic and extradendritic flow.

arm spacing of $20 \mu \mathrm{m}$ ), and grain size (10 different values assuming a cooling rate of $10 \mathrm{~K} / \mathrm{s}$ ), using a domain containing 8000 realistic grains with an average grain size of $300 \mu \mathrm{m}$, to provide over 600 unique permeability values. and deformation $\left(\beta=0, \dot{\varepsilon}_{s v}=0\right)$.

Fig. 8 provides two permeability maps that show the range of solid fraction where the Carman-Kozeny equation with Dendritic $S_{v}$ or Globular $S_{v}$ is valid in predicting permeability; (a) as a function of cooling rate and (b) as a function of dimensionless grain size $d /\left(2 \cdot \lambda_{2}\right)$. Each map is divided into two shaded areas corresponding to Globular $S_{v}$ and Dendritic $S_{v}$, and an unshaded area where neither expression matches the simulation result within the tolerance of $50 \%$. Beginning with Fig. 8(a) it can be seen that under conditions of lower cooling rates the CarmanKozeny equation with Globular $S_{v}$ is most appropriate; the valid solid fraction range will decrease with increasing cooling rate and is no longer valid once the cooling rate exceeds $15 \mathrm{~K} / \mathrm{s}$. At high cooling rates especially greater than $10 \mathrm{~K} / \mathrm{s}$, the Carman-Kozeny equation with Dendritic $S_{v}$ is most appropriate over a wide range of solid fraction. However, at low solid fraction there are no circumstances where the simulated permeability matches the analytical expressions. Further, there is an important combination of cooling rate and solid fraction where neither analytical expression is valid, covering all solid fractions. Any macroscale model, having the same solidification conditions, and utilizing the Carman-Kozeny equation with one of these two limiting cases could show discrepancies as compared to experimental findings. Turning now to Fig. 8(b), it can be seen that the Carman-Kozeny equation with Dendritic $S_{v}$ or Globular $S_{v}$ is no longer valid when $d /\left(2 \cdot \lambda_{2}\right)$ is greater than 30 for the specific cooling rate of $10 \mathrm{~K} / \mathrm{s}$. At lower values of this dimensionless grain size the Carman-Kozeny expression for globular structures is found to be valid at very high solid fractions as the liquid is mostly dominated 
by the extra-dendritic flow whereas the Carman-Kozeny expression for dendritic structures is only valid over a very 514 small range of parameters.
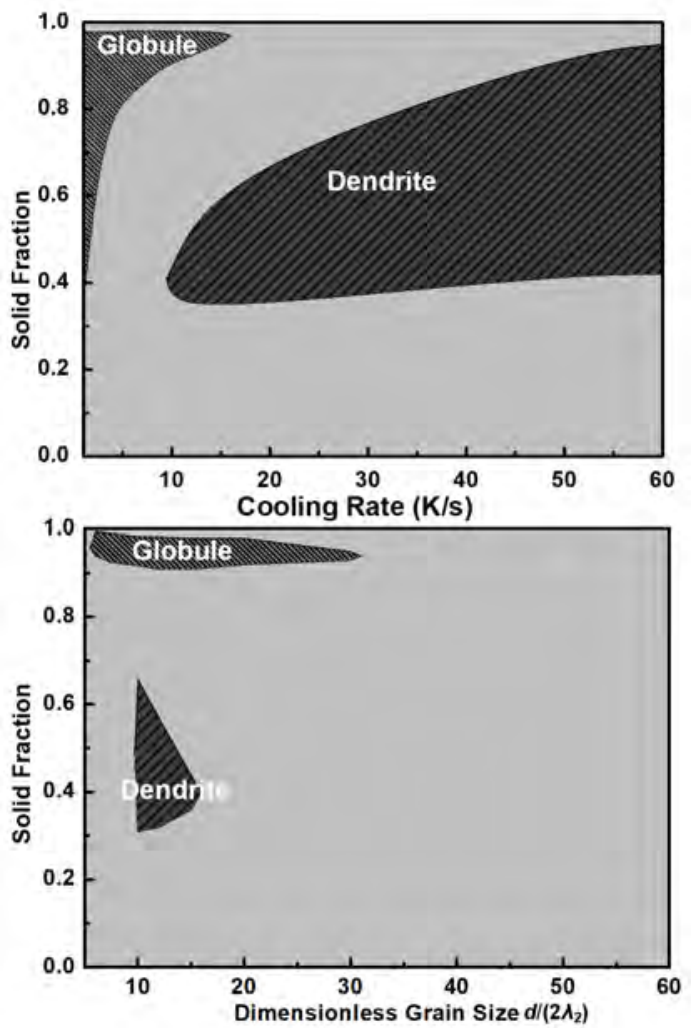

Figure 8: Permeability map as a function of solid fractions and (a) cooling rate as well as (b) dimensionless grain size, $d /\left(2 \cdot \lambda_{2}\right)$.

It is clear that from Figs. 6 to 8 that the permeability of a semisolid domain containing a mixture of two morpholo- ${ }^{54}$ gies cannot be predicted with the Carman-Kozeny equation via a single scaling law for $S_{v}$ throughout the whole ${ }^{548}$ solid fraction range. Although this is commonly done in macrosegregation simulations, it makes the permeability assessment less accurate. Utilizing our 3D equiaxed- ${ }^{55}$ dendritic meso-scale solidification model [19, it is possible to calculate $S_{v}$ for a domain containing multiple morpholo-- ${ }^{552}$ gies as

$$
S_{v}=\frac{\sum_{i=1}^{N_{\text {elem }}} S_{s l}^{e}}{V_{\text {domain }}},
$$
with $S_{s l}^{e}=\left\{\begin{array}{l}\lambda_{2} \\ S_{\text {globule }}, g_{s}^{\prime} \geq g_{\text {critical }}^{\prime}\end{array}\right.$ where $N_{\text {elem }}$ represents the total number of elements within the domain.

$S_{s l}^{e}$ represents the solid/liquid interfacial area of an indi-562 vidual element, $V_{\text {domain }}$ is the total volume of the domain,

$V_{e n v}$ is the volume of the dendrite envelope and $S_{\text {globule }}$ is564 the surface area of globular element.

The key point in determining the $S_{v}$ through our solid-566 ification model is identification of the internal solid fraction $g_{s}^{\prime}$ at which flow within an individual element is dom-568 inated by intra-dendritic or extra-dendritic character; if the $g_{s}^{\prime}$ is greater than critical point $g_{\text {critical }}^{\prime}$ the element is treated to as globular and intra-dendritic liquid flow is ignored. Fig. 9 plots the permeability calculated from the Carman-Kozeny equation utilizing the solidification model -calculated $S_{v}$ for five different critical values of $g_{s}^{\prime}$, as well as the prediction from the 3D fluid flow model for a cooling rate of $5 \mathrm{~K} / \mathrm{s}$, and the phenomenological macroscale model developed by Wang et al 20]. As can be seen, the approach to calculate $S_{v}$ via our solidification model results in a clear transition zone in permeability from dendritic to globular character and matches much more closely to the model-predicted value than the Dendritic $S_{v}$ and Globular $S_{v}$ cases, and Wang's model. However, deviations still exist and the importance of selecting the "right" value of $g_{s}^{\prime}$ is evident since a higher critical value of $g_{s}^{\prime}$ provides smaller deviation at higher solid fractions but then under-estimates the permeability at lower solid fraction. The determination of the critical point of $g_{s}^{\prime}$ requires further investigations both experimentally and numerically. The observed differences could also be due to limitations within the 3D fluid flow model, which is built on the following assumptions: a uniform porous medium within the dendrite envelope with locally $S_{v}=\frac{2}{\lambda_{2}}$ and Poiseuille flow when $g_{s}^{\prime} \sim 0$. Another option for overcoming the limitations of using $S_{v}$ calculated by $\lambda_{2}$ would be to use a general form that considers grain growth, coalescence and impingement 2].

It should be noted that Wang's model also shows a transition between the two limiting cases, but the overall permeability predicted by this phenomenological approach matches with the Carman-Kozeny results for globular $S_{V}$ at a much lower solid fraction $\sim 0.68$ than our model-predicted values. This is due to the simplified assumption of average grain size used in Wang's model which fails to consider the influence of grain size distribution and thus the flow path within different grains.

\subsubsection{Localization of Liquid Feeding}

In a domain that contains different grain sizes, different semisolid morphologies are possible as shown in Fig. 6(b). Due to these different morphologies, flow is likely to concentrate in areas with a higher local permeability. To reproduce this feeding localization, a set of simulations were carried out by imposing a pressure difference between the top and bottom surfaces of the domain consisting of 8000 realistic grains, $p_{0}=0 \mathrm{~Pa}$ and $p_{1}=-2 \mathrm{MPa}$, while the lateral surfaces were closed, and solidification shrinkage and deformation were neglected $\left(\beta=0, \dot{\varepsilon}_{s v}=0\right)$. These conditions provide uni-directional flow with the same flow rate of liquid entering and leaving the domain.

Fig. 10 shows the 3D permeability map and corresponding local fluid velocity resulting from these simulations at solid fractions of (a) 0.70 and (b) 0.84 to highlight the capability of the fluid flow model in predicting the localization of liquid feeding. First, by examining a1 , it can be seen that the permeability between different 


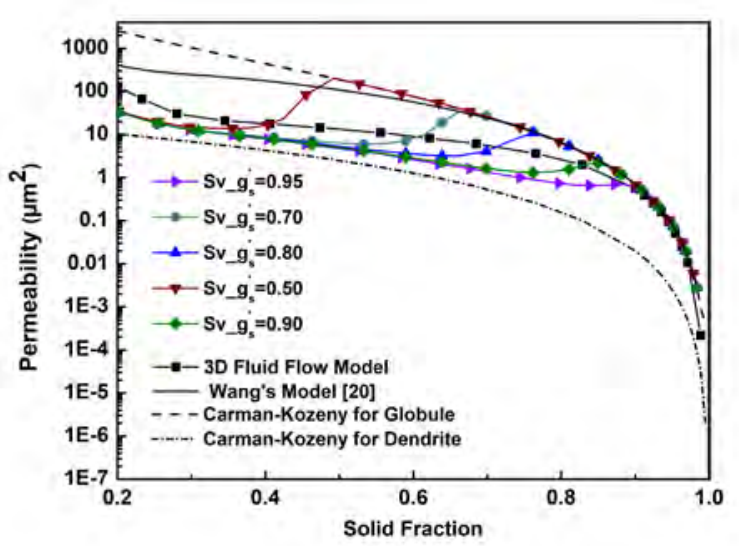

Figure 9: Influence of $S_{v}$ calculated based on different internal solid fractions on the prediction of the permeability within mushy zone618 via Carman-Kozeny equation. The permeability calculated via the Carman-Kozeny for Globular and Dendritic cases, as well as via Wang's model 20] is provided for comparison purposes. Wang's ${ }^{620}$ model was applied using a grain size of $300 \mu \mathrm{m}$ and a dendrite envelope sphericity $\phi_{e}=1$.

grains varies considerably, due to differences in $g_{s}^{\prime}$ and the extra-dendritic liquid channel width. The maximum local permeability at bulk $g_{s}=0.7$ is $10730 \mu \mathrm{m}^{2}$; the value of $0 \mathrm{\mu m}^{2}$ represents grains that have fully solidified. As the permeability for globular structures is higher than den-622 dritic structures at the same solid fraction (Fig. 7), this variation in local permeability would lead to further lo-624 can be seen that the fluid selectively flows through areas626 having larger local permeability, at higher local speeds. At higher solid fraction, $g_{s}=0.84$ and shown in a-2, the max-628 imum local permeability decreases to $4096 \mathrm{\mu m}^{2}$ due to the increase in $g_{s}^{\prime}$ and narrowing of the extra-dendritic liquid630 channels. The maximum liquid channel velocity, shown in b-2, consequently also decreases.

\subsection{Fluid flow induced by phase changes and tensile de-634 formation}

The 3D dendritic fluid flow model can also be used ${ }^{636}$ to calculate the amount of liquid required to compensate phase changes and imposed tensile deformations under ${ }^{638}$ dendritic solidification conditions. This requires activation of the shrinkage and deformation terms of Eq. $15\left(i . e^{640}\right.$ $\beta \neq 0, \dot{\varepsilon}_{s v} \neq 0$ follow Eqs. 9 and 13 Four different compositions were assessed; Fe-0.07wt.\% (non-peritectic), $\mathrm{Fe}^{-{ }^{642}}$ 0.12 wt.\%C (hypo-peritectic), Fe-0.16wt.\%C (peritectic) and Fe-0.18wt.\%C (hyper-peritectic). The solidification simu- ${ }^{644}$ lations contained 8000 cubic grains, $500 \mu \mathrm{m}$ in size, cooled at a rate of $55 \mathrm{~K} / \mathrm{s}$. The uniform selection of grain size $\left(\mathrm{cu}^{-646}\right.$ bic grain) and the high cooling rate ensured the creation of a fully dendritic semisolid structure. For boundary con- ${ }^{648}$ ditions, the flow simulation assumed that all the domain surfaces except the one on the top were closed and a gauge ${ }^{650}$ pressure of $0 \mathrm{~Pa}$, was imposed on the top surface. Hence, the liquid suction from top surface due to shrinkage and deformation can be predicted.

Fig. 11(a) shows the variation in net liquid flow per unit volume $(Q / V)$ predicted by the 3D dendritic fluid flow model to compensate for solidification shrinkage in all four of the carbon compositions of interest assuming $\beta \neq 0$ and $\dot{\varepsilon}_{s v}=0$. First, as expected, it can be seen that although the predicted inflow of liquid decreases with increasing solid fraction, the net liquid flow is significantly different dependent on alloy composition. Interestingly, a sharp rise in net fluid flow is predicted to be needed to compensate for shrinkage in the peritectic alloy once the peritectic transformation starts to account for the additional density difference of the austenitic phase. The sharp rise occurs at a relatively low solid fraction for the hyper-peritectic alloy, followed by the peritectic and hypoperitectic alloy at increasing $g_{s}$. The net fluid flow required then remains relatively constant until the final stages of solidification.

For a non-peritectic alloy, the amount of liquid required to compensate for solidification shrinkage can also be calculated analytically as

$$
\left(\frac{Q}{V}\right)=\beta \frac{d g_{s}}{d t},
$$

where $Q$ and $V$ represent the volumetric flow rate and total domain volume. For further validation purposes, the shrinkage calculated by this equation for a Fe- 0.07 wt.\%C alloy is also shown in Fig. 11(a). As can be seen, a good match is obtained between the simulation and analytical curves.

The amount of liquid required during solidification and peritectic transformation can be linked to the formation of casting defects. Liquid flow that is inadequate to compensate for the solidification shrinkage could result in the formation of large voids to maintain continuity. At low solid fraction, a high permeability likely allows for adequate liquid feeding to heal any formed defects. At high solid fraction, Fig. 11(a) shows that for the hyper-peritectic alloy, the jump in fluid required due to the peritectic transformation occurs at a "low-enough" solid fraction where the permeability remains relatively high. Using the same argument, defects would be most prone to occur in the hypoperitectic alloy (Fe- $0.12 \mathrm{wt} . \% \mathrm{C})$ since the peritectic transformation occurs at a very high solid fraction where the permeability is quite low (Fig. 11(a)). Pressure contours of hypo-peritectic alloy are also plotted for different solid fractions to emphasis the influence of peritectic transformation shown in Fig. 11(b). It can be seen that before peritectic transformation, an increase in solid fraction would result in a minor increase in the pressure drop by comparing Fig. 11(b1) and (b2), while a significant pressure drop occurs after the peritectic transformation as shown in Fig. 11(b3) which is two orders of magnitude greater than Fig. 11(b1) and (b2). The high pressure drop near the end of solidification would accelerate the formation of 

been reported in prior work 28

Liquid feeding can be also induced by the deformation of the mushy zone. If, concurrently, semisolid tensilez10 hot tear will form. Generally, the amount of net inflow 712

of liquid required during solidification is a given by the combination of shrinkage and deformation. In order tor14 investigate the dominant factors that cause hot tearing in hypo-peritectic grades (Fe-0.12wt.\%C), known to be most-716 sensitive to hot tearing [28], a series of simulations were performed that consider both shrinkage and deformation 718 ( $\beta \neq 0$ and $\dot{\varepsilon}_{s v} \neq 0$ ); the same boundary conditions as for Fig. 11 were utilized.

Fig. 12 shows the net flow caused by the combination of solidification shrinkage and deformation, and their contributions under two different strain rates of $0.1 \mathrm{~s}^{-1}$ and $0.001 \mathrm{~s}^{-1}$. Under the strain rate of $0.1 \mathrm{~s}^{-1}$, the in- ${ }_{722}$ duced liquid feeding mainly comes from deformation at lower solid fractions $(<0.92)$, and amount of liquid required would increase when the peritectic transformation occurs mentioned in the prior section. The dominant factor near the end of solidification would due to the large amount of shrinkage caused by the peritectic transformation.

Under small strain rate of $0.001 \mathrm{~s}^{-1}$ also shown in Fig 12(lower), clearly, lower strain rates result in a less liq-728 uid flow to counteract deformation. The net flow caused by shrinkage and deformation is dominated by the solid- ${ }^{730}$ ification shrinkage. In the industrial process, the $\operatorname{strain}_{732}$ rates during casting of steel are thought to be relatively small, on the order of $10^{-3} \sim 10^{-4} \mathrm{~s}^{-1}$. The results shown ${ }^{734}$ in Fig. 12 then seem to indicate that shrinkage associated ${ }_{736}$ with the large interfacial area of the dendritic structure is the key factor to cause defects.

\section{Conclusions}

A 3D dendritic fluid flow model has been developed to quantitatively predict the fluid flow behaviour induced ${ }^{744}$ by the solidification shrinkage at the meso-scale, through ${ }_{746}$ thousands of equiaxed grains. The model is based on the Darcy-Brinkman form of the Navier-Stokes equation at $^{748}$ a prescribed solid fraction. Using the framework of the ${ }_{750}$ Voronoi tessellation, the tortuosity of flows around the complex interdendritic channels was considered. This new ${ }^{752}$ technique captures both semi-solid morphology and the fluid flow behavior during solidification, and provides an ${ }^{754}$ alternative to the convectional experiment for the predic-756 tion of permeability by using the given surface area concentration. Comparison of the numerical and experimen- ${ }^{-758}$ tal permeabilities shows a good agreement (within $\pm 5 \%)_{760}$ for either extra-dendrite or intra-dendritic flow, and deviation from the conventional Carman-Kozeny equations ${ }^{762}$ using simplified Dendritic $S_{v}$ or Globular $S_{v}$ are explained in detail. The results quantitatively demonstrate the ef- ${ }^{764}$ fect of grain size and microstructure morphology during solidification on the permeability prediction.
The localization of liquid feeding under the pressure gradient is also reproduced in the present investigation. The results highlight the ability in predicting liquid feeding within a semisolid domain where local permeability varies. Additionally, the advection of fluid due to shrinkage and deformation for non-peritectic and peritectic steel grades with dendritic morphology during solidification was captured for the first time, and the results were validated with empirical equations. Due to the large solid/liquid surface area of the dendritic structure, the advection of fluid is dominated by the shrinkage during the peritectic phase transformation within the mushy zone under the small deformation rate, and easily cause the formation of casting defect.

\section{Acknowledgements}

The authors wish to thank the Natural Sciences and Engineering Research Council of Canada (NSERC) and the McMaster Steel Research Centre for financial support.

\section{References}

[1] M. El-Bealy, B. G. Thomas, Prediction of dendrite arm spacing for low alloy steel casting processes, Metallurgical and materials transactions B 27 (4) (1996) 689-693.

[2] H. Neumann-Heyme, K. Eckert, C. Beckermann, General evolution equation for the specific interface area of dendrites during alloy solidification, Acta Materialia 140 (2017) 87-96.

[3] M. Rappaz, J.-M. Drezet, M. Gremaud, A new hot-tearing criterion, Metallurgical and materials transactions A 30 (2) (1999) 449-455.

[4] E. Khajeh, D. M. Maijer, Physical and numerical characterization of the near-eutectic permeability of aluminum-copper alloys, Acta Materialia 58 (19) (2010) 6334-6344.

[5] D. Bernard, Ø. Nielsen, L. Salvo, P. Cloetens, Permeability assessment by $3 \mathrm{~d}$ interdendritic flow simulations on microtomography mappings of al-cu alloys, Materials Science and Engineering: A 392 (1-2) (2005) 112-120.

[6] T. Takaki, S. Sakane, M. Ohno, Y. Shibuta, T. Aoki, Permeability prediction for flow normal to columnar solidification structures by large-scale simulations of phase-field and lattice boltzmann methods, Acta Materialia 164 (2019) 237-249.

[7] E. Khajeh, D. Maijer, Numerical determination of permeability of al-cu alloys using 3d geometry from x-ray microtomography, Materials Science and Technology 26 (12) (2010) 1469-1476.

[8] E. Khajeh, D. M. Maijer, Permeability of dual structured hypoeutectic aluminum alloys, Acta Materialia 59 (11) (2011) $4511-4524$.

[9] E. Khajeh, D. M. Maijer, Permeability evolution during equiaxed dendritic solidification of al-4.5 wt\% cu, Modelling and Simulation in Materials Science and Engineering 20 (3) (2012) 035004.

[10] D. Fuloria, P. D. Lee, D. Bernard, Microtomographic characterization of columnar al-cu dendrites for fluid flow and flow stress determination, Materials Science and Engineering: A 494 (1-2) (2008) 3-9.

[11] C. Puncreobutr, P. Lee, K. Kareh, T. Connolley, J. Fife, A. Phillion, Influence of fe-rich intermetallics on solidification defects in al-si-cu alloys, Acta Materialia 68 (2014) 42-51.

[12] S. Vernède, J. A. Dantzig, M. Rappaz, A mesoscale granular model for the mechanical behavior of alloys during solidification, Acta Materialia 57 (5) (2009) 1554-1569. 
13] M. Sistaninia, A. Phillion, J.-M. Drezet, M. Rappaz, A 3-d coupled hydromechanical granular model for simulating the constitutive behavior of metallic alloys during solidification, Acta Materialia 60 (19) (2012) 6793-6803.

[14] S. Vernede, P. Jarry, M. Rappaz, A granular model of equiaxed mushy zones: Formation of a coherent solid and localization of feeding, Acta Materialia 54 (15) (2006) 4023-4034.

15] M. Sistaninia, A. Phillion, J.-M. Drezet, M. Rappaz, Threedimensional granular model of semi-solid metallic alloys undergoing solidification: Fluid flow and localization of feeding, Acta Materialia 60 (9) (2012) 3902-3911.

[16] H. Z. Rajani, A. Phillion, 3d multi-scale multi-physics modelling of hot cracking in welding, Materials \& Design 144 (2018) 45-54.

[17] J. Ni, C. Beckermann, A volume-averaged two-phase model for transport phenomena during solidification, Metallurgical Transactions B 22 (3) (1991) 349

[18] D. Tourret, C.-A. Gandin, A generalized segregation model for concurrent dendritic, peritectic and eutectic solidification, Acta Materialia 57 (7) (2009) 2066-2079.

[19] Y. Feng, A. Phillion, A 3d meso-scale solidification model for metallic alloy using a volume average approach, Materialia 6 (2019) 100329.

[20] C. Wang, S. Ahuja, C. Beckermann, H. De Groh, Multiparticle interfacial drag in equiaxed solidification, Metallurgical and Materials Transactions B 26 (1) (1995) 111-119.

21] M. Le Bars, M. G. Worster, Interfacial conditions between a pure fluid and a porous medium: implications for binary alloy solidification, Journal of Fluid Mechanics 550 (2006) 149-173.

[22] P. C. Carman, Fluid flow through granular beds, Trans. Inst. Chem. Eng. 15 (1937) 150-166.

[23] J. Bear, Dynamics of fluids in porous media, Courier Corporation, 2013

[24] J. A. Dantzig, M. Rappaz, Solidification: -Revised \& Expanded, EPFL press, 2016.

[25] H. Mizukami, A. Yamanaka, Generation mechanism of unevenness of ultra low carbon steel at initial stage of solidification, ISIJ international 50 (3) (2010) 435-444.

[26] J. Dongarraxz, A. Lumsdaine, X. Niu, R. Pozoz, K. Remingtonx, A sparse matrix library in $\mathrm{c}++$ for high performance architectures, in: Second object oriented numerics conference, Citeseer, 1994, pp. 214-218.

[27] L. Zhang, S. Taniguchi, K. Cai, Fluid flow and inclusion removal in continuous casting tundish, Metallurgical and Materials Transactions B 31 (2) (2000) 253-266.

[28] P. Presoly, R. Pierer, C. Bernhard, Identification of defect prone peritectic steel grades by analyzing high-temperature phase transformations, Metallurgical and Materials Transactions A 44 (12) (2013) 5377-5388.

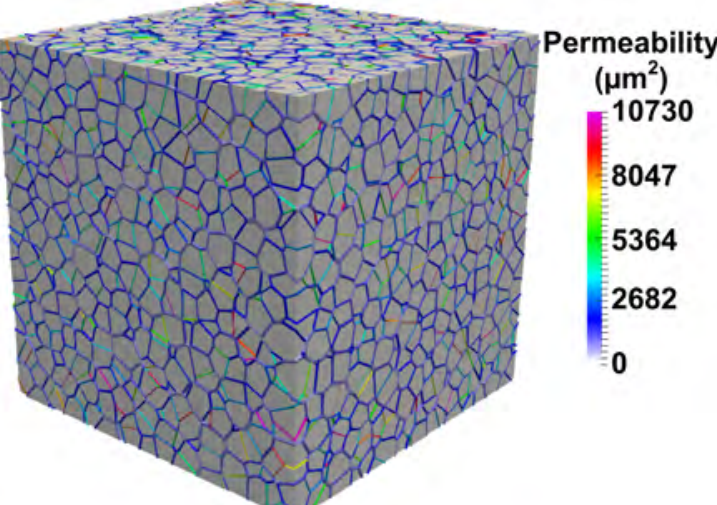

(a1)

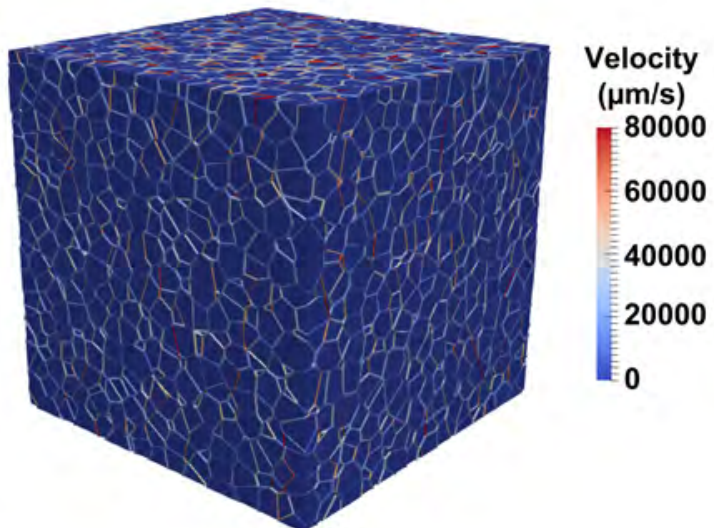

(a2)

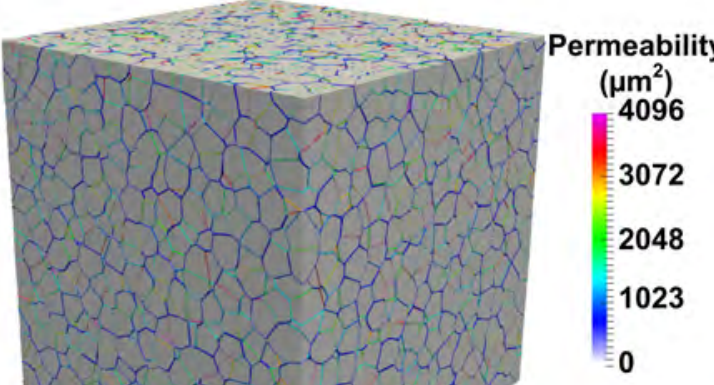

0

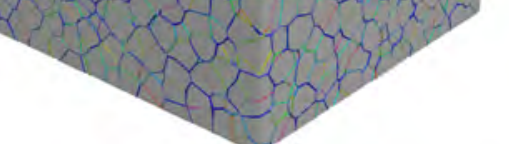

(b1)

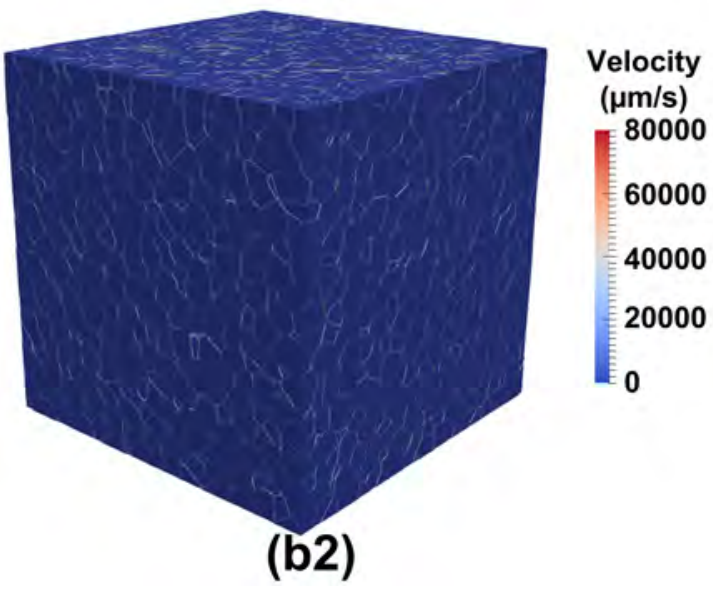

Figure 10: Variations in permeability $(-1)$ and local velocity $(-2)$

11 within a semisolid domain at (a) $g_{s}=0.70$ and (b) $g_{s}=0.84$. The grain size was $500 \mu \mathrm{m}$, and the cooling rate was $5 \mathrm{~K} / \mathrm{s}$ 


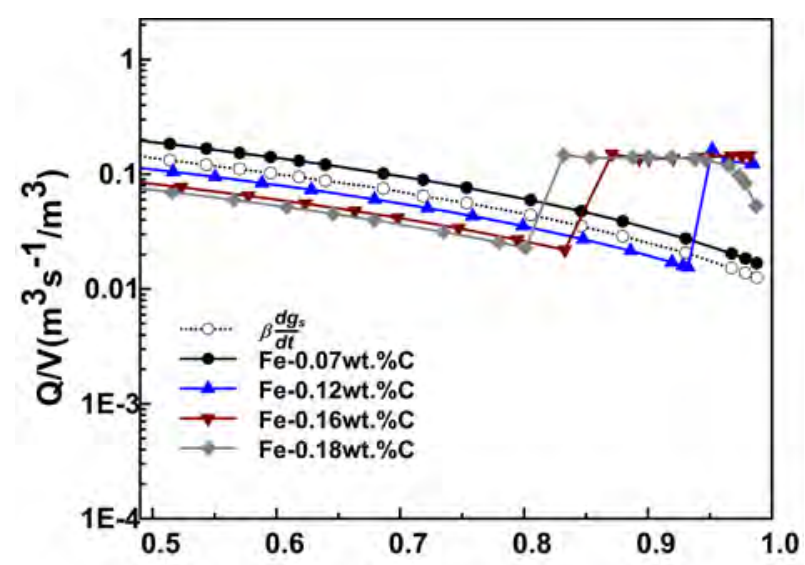

(a) Solid Fraction

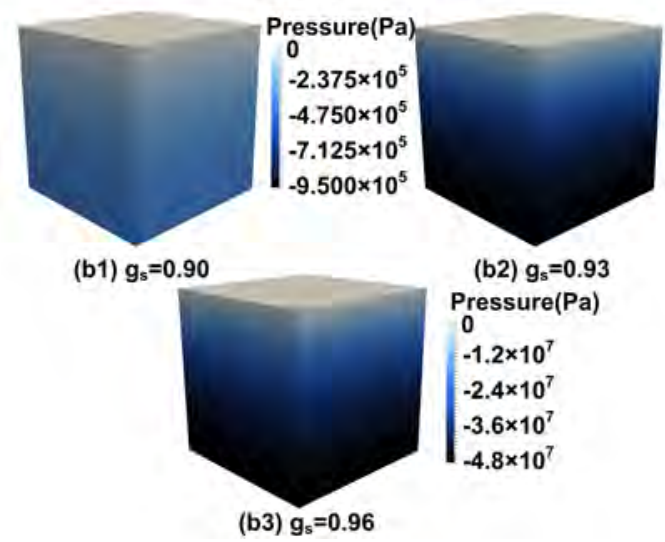

Figure 11: A comparison of $Q / V$ predicted by the 3D dendritic fluid flow model and Eq. 20 as a function of solid fraction for various $\mathrm{Fe}-$ $\mathrm{C}$ alloys along with the pressure contours at three solid fractions for Fe- 0.12 wt. \% alloy. The required flux to compensate for the peritectic transformation in peritectic grades is also included in the flow predictions of the 3D dendritic fluid flow model. Note that Fig. 10(b1) and (b2) share the same color bar.
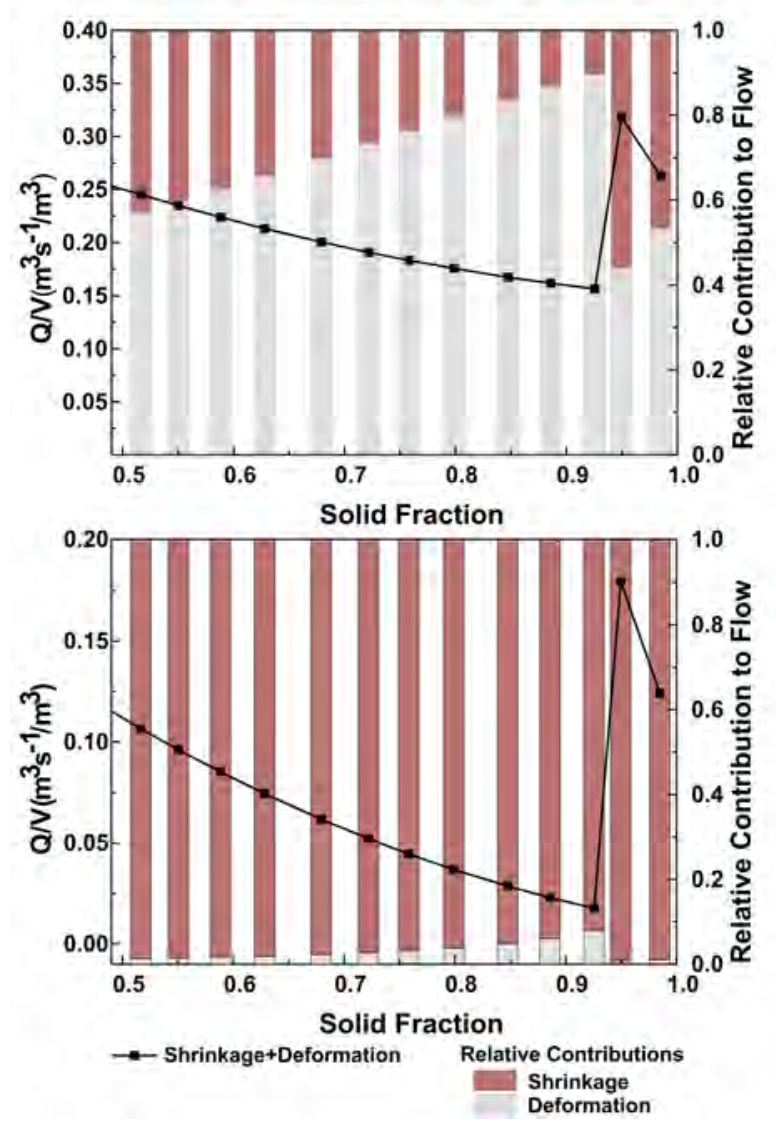

Figure 12: A comparison of the $Q / V$ predicted by the 3D dendritic fluid flow model taking into account both solidification shrinkage and deformation. Strain rates of $0.1 \mathrm{~s}^{-1}$ (upper) and $0.001 \mathrm{~s}^{-1}$ (lower) are examined. 